\title{
Age-Specific Effectiveness and Safety of Newly Initiated Insulin Therapy in Japanese Patients with Uncontrolled Diabetes
}

\author{
Takayoshi Shiga $\cdot$ Hirohide Yokokawa $\cdot$ Yoshinobu Taneda • \\ Eiichiro Sugihara $\cdot$ Mayumi Meijyo $\cdot$ Kazunori Mitsuhashi $\cdot$ \\ Teruhiko Hisaoka $\cdot$ Hiroshi Isonuma
}

To view enhanced content go to www.diabetestherapy-open.com Received: October 22, 2013 / Published online: December 10, 2013

(c) The Author(s) 2013. This article is published with open access at Springerlink.com

\section{ABSTRACT}

Introduction: One consequence of population aging is an increase in the number of elderly patients with diabetes mellitus. These elderly patients often experience atherosclerotic complications, and diabetes prevention and management are strongly desired to promote health and reduce the financial burden on the healthcare system. In this study, we conducted an age-specific evaluation of the effectiveness and safety of comprehensive management with

Electronic supplementary material The online version of this article (doi:10.1007/s13300-013-0049-4) contains supplementary material, which is available to authorized users.

T. Shiga $\cdot$ H. Yokokawa $(\bowtie) \cdot$ E. Sugihara

K. Mitsuhashi · T. Hisaoka · H. Isonuma Department of General Medicine, Juntendo University School of Medicine, Hongo 2-1-1, Bunkyo-ku, Tokyo 113-8421, Japan e-mail: yokokawa@pa3.so-net.ne.jp

Y. Taneda $\cdot$ M. Meijyo

Taneda Medical Clinic, Fukushima, Japan

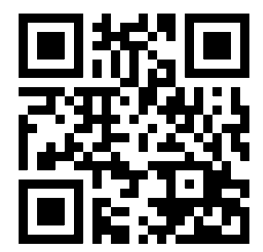

Enhanced content for this article is available on the journal web site: www.diabetestherapy-open.com newly initiated insulin therapy over a 1-year period in elderly ( $\geq 65$ years) compared with non-elderly ( $\leq 64$ years) Japanese patients with uncontrolled diabetes [glycated hemoglobin (HbA1c) $\geq 8 \%$ for $\geq 3$ months].

Methods: This retrospective single-center cohort study was conducted in Japan. We screened all outpatients with diabetes mellitus who visited the clinic for diabetes treatment between December 2006 and March 2011. Of these patients, 132 with type 2 diabetes who were newly initiated on insulin therapy for continued poor glycemic control and undergoing comprehensive management through self-monitoring of blood glucose (SMBG) were registered to the study.

Results: Thirty-two of 132 registered patients were excluded from the analysis. Among the 100 patients (67 non-elderly, 33 elderly) included in the analysis, median age and proportion of male patients was 69 years and $66.7 \%$, respectively, among the elderly, and 52 years and $68.7 \%$, respectively, among the non-elderly patients. After initiation of insulin therapy, median HbA1c levels improved from $9.6 \%$ to $7.2 \%$ in elderly patients, and from $10.8 \%$ to $7.3 \%$ in non-elderly patients at 
baseline and 12 months. Severe hypoglycemic events were not observed in either patient group; however, uncontrolled diabetes was ongoing in $31.8 \%$ of non-elderly and $15.4 \%$ of elderly patients, and obesity was associated with poor glycemic control.

Conclusion: Our results indicate that the effectiveness and safety of newly initiated insulin therapy are similar between elderly and non-elderly Japanese patients with uncontrolled diabetes, and highlight the importance of comprehensive management using SMBG to avoid hypoglycemia. Better glycemic control supported by adequate intensive management is required to improve mortality and morbidity.

Keywords: Diabetes mellitus; Elderly; Insulin therapy; Management; Self-monitoring of blood glucose

\section{INTRODUCTION}

Populations are rapidly aging worldwide. This is particularly true in Japan, where there is an increased need to address problems associated with the aging population $[1,2]$. One consequence of population aging is an increase in the number of elderly patients with diabetes mellitus [glycated hemoglobin A1c $(\mathrm{HbA1c}) \geq 6.5 \%$ ] [3]. A Japanese nationwide survey reported an increase in the prevalence of diabetes mellitus with advancing age, and diabetes mellitus was suspected in approximately $23 \%$ of men and $12 \%$ of women aged $\geq 60$ years [3]. These patients often have atherosclerotic complications $[4,5]$, and diabetes prevention and management are strongly desired to promote health and reduce the impact on healthcare expenditure.

Diabetes treatment guidelines that recommend intensive multifactorial interventions to prevent macro- and microcomplications have been established for young and middle-aged patients with diabetes [5]. However, treatment strategies for elderly patients with diabetes are not well established, and treatment goals for glycemic control are controversial because of insufficient accumulated clinical evidence and difficulties associated with intensive therapy in elderly patients [4-6]. Of particular concern, intensive treatment in elderly patients with diabetes may increase risk of death [7], falls [8], and cognitive function decline [9] due to hypoglycemic events. Therefore, glycemic control targets should depend on individual patient characteristics, for example, the newest position statement recommends lower glycemic control targets (HbA1c $<6.5-7.0 \%$ ) for healthy elderly patients, while an HbA1c $<7.0-8.0 \%$ may be acceptable if lower targets cannot be achieved [6].

Regardless of these concerns, comprehensive management to prevent atherosclerotic complications requires the achievement of adequate glycemic control in elderly as well as non-elderly patients with diabetes $[4,5]$. Unfortunately, only a few studies have evaluated the safety and efficacy of comprehensive management in elderly patients [10]. The current study was an agespecific evaluation of the effectiveness and safety of newly initiated insulin therapy over a 1 -year period in elderly ( $\geq 65$ years) and nonelderly ( $\leq 64$ years) Japanese patients with uncontrolled diabetes, incorporating comprehensive management with SMBG.

\section{MATERIALS AND METHODS}

\section{Patients}

The present study was a retrospective cohort study conducted at a specialist medical clinic 
for diabetes treatment in Fukushima, Japan. We surveyed all outpatients with diabetes who visited the clinic for diabetes treatment from December 2006 to March 2011. Study participants were then selected from those patients who were initiated on insulin therapy during the study period, including those whose medication was modified from oral hypoglycemic agents to insulin therapy and those who were prescribed insulin as add-on therapy due to continued poor glycemic control, defined as HbA1c [National Glycohemoglobin Standardization Program (NGSP)] [11] $\geq 8.0 \%$ for at least 3 months [calculated using the following formula: HbA1c $\quad($ NGSP,$\%)=1.02 \times$ HbA1c $\quad$ Japan Diabetes Society [JDS]) $+0.25 \%$ ]. Patients with concomitant chronic diseases such as kidney dysfunction (plasma creatinine $\geq 2 \mathrm{mg} / \mathrm{dL}$ ), severe cardiovascular disease, severe cognitive dysfunction, poor nutritional condition, and type 1 diabetes mellitus were excluded from the study.

There were three certified diabetes educators and one dietitian in the medical clinic. The attending physician determined the patients' insulin therapy strategies, taking into consideration diabetic control status, comorbidities, and family support. A certified diabetes educator then met with the patient to instruct him or her in the use of insulin devices and SMBG. Patients were instructed to measure their blood glucose concentration twice each day (before breakfast and dinner). After initiation of insulin therapy, and at monthly medical consultations, the attending physician checked the patient's HbA1c levels and SMBG records, and modified their medications as necessary. The certified diabetes educator also checked the use of insulin devices and SMBG procedures every 2 or 3 months. The dietitian met all patients to evaluate their dietary behavior and to provide diabetic dietary advice once or twice a year.

\section{Variables}

Baseline clinical data at initiation of insulin therapy were obtained from the patients' medical files. Data collection was conducted from November 2012 to February 2013 by three office staff at the medical clinic. The following demographic data were collected: age, sex, height, weight, family history (hypertension, diabetes mellitus, heart disease, and cerebrovascular disease), presence of micro- and macrovascular diseases [diabetic neuropathy (sensory symptoms present, absent ankle reflexes, inability to sense vibration), diabetic retinopathy, diabetic nephropathy (present micro- and/or macroalbuminuria), cardiovascular disease, and cerebrovascular disease], alcohol consumption, and smoking status. To assess blood pressure related items, we collected information on systolic and diastolic blood pressure, and medication history for hypertension. We also collected total cholesterol (mg/dL; TC), high-density lipoprotein cholesterol (mg/dL; HDL-C), lowdensity lipoprotein cholesterol (mg/dL; LDL-C), triglyceride (mg/dL; TG), and medication history for hyperlipidemia. LDL-C was estimated using the Friedwald equation [(TC) - (HDL-C) - (TG/ 5)] [12]. These were baseline data before initiation of insulin therapy, and also collected retrospectively from medical files.

The following diabetes-related information was collected as baseline data: just the latest HbA1c measurement before initiation of insulin therapy, type of insulin initiated, insulin dosage per day, and type of oral hypoglycemic agent used. HbA1c levels were determined by highperformance liquid chromatography using an automated analyzer (Arkray, Kyoto, Japan). 
HbA1c was measured and hypoglycemic events were checked every month. We collected follow-up data from medical records: HbA1c measurements at 3, 6, and 12 months after initiation of insulin therapy, including bodyweight change and insulin dosage at 12 months, and adverse effects including severe hypoglycemic events and withdrawal from insulin therapy. Body weight also measured every month, and lipid-related measurements were measured every 3 or 4 months. In the analysis, we used baseline data and data at 12 months.

This survey was conducted according to the Ethical Guidelines for Epidemiological Studies established by the Japanese government [13], and with the Helsinki Declaration of 1975, as revised in 2000 and 2008. The Ethics Committee of Juntendo University approved the research protocol (No. 832). Informed consent was obtained from all patients for being included in the study.

\section{Statistical Analysis}

Primary endpoint of the study was HbA1c levels after initiation of insulin therapy and secondary endpoint was severe hypoglycemic complications. Participants were categorized into two age categories: elderly patients ( $\geq 65$ years) and non-elderly patients ( $\leq 64$ years). The Mann-Whitney test for continuous variables and the Chi-square test for categorical variables were used to compare the two groups. For paired analysis, the Wilcoxon signed-rank test was used for continuous variables. $P<0.05$ was considered statistically significant. All statistical analyses were performed using the Statistical Package for Social Sciences version 19 (SPSS Inc., Chicago, IL, USA).

\section{RESULTS}

\section{Patients}

During the study period, 132 patients with type 2 diabetes began insulin therapy at the medical clinic. Thirty-two of these patients were excluded from the current study based on exclusion criteria (4), lack of data (13), or were lost to follow-up (15). The remaining 100 patients were enrolled in the study. Baseline characteristics of these participants are shown in Table 1.

Of the 100 participants, 33 (33.0\%) were elderly ( $\geq 65$ years) and 67 (67.0\%) were nonelderly. Median age among the elderly and nonelderly patients was 69 years (range 65-84) and 52 years (range 19-64; $P<0.01$ ), respectively. The proportions of male patients were $66.7 \%$ and $68.7 \%$, respectively. Median BMIs of the elderly and non-elderly patients were $23.0 \mathrm{~kg} /$ $\mathrm{m}^{2}$ (19.2-29.5) and $24.0 \mathrm{~kg} / \mathrm{m}^{2}$ (18.0-35.8), respectively. Antihypertensive drug use was significantly more frequent $(57.6 \%$ vs $26.9 \%$, $P<0.01)$ and diabetic neuropathy was more common $(75.8 \%$ vs $46.3 \%, P<0.01)$ among elderly compared to non-elderly patient.

At baseline, elderly patients were prescribed sulfonylureas and biguanides to treat diabetes more frequently than non-elderly patients (Table 1). After initiation of insulin therapy, non-elderly patients were more likely to be treated with ultra-rapid insulin $(62.7 \%$ vs $15.2 \%, P<0.01)$, while elderly patients were more likely to be treated with long-acting insulin $(20.9 \%$ vs $60.6 \%, P<0.01)$. No severe hypoglycemic complications were observed in either group during the study period; insulin therapy was stopped in 3 elderly (9.1\%) and 10 non-elderly (14.9\%) patients because of improvements in their glycemic control. 
Table 1 Baseline characteristics of the study participants $(N=100)$

\begin{tabular}{|c|c|c|c|}
\hline & \multicolumn{2}{|l|}{ Median (range) or $N(\%)$} & \multirow[t]{2}{*}{$P$ value } \\
\hline & $\begin{array}{l}\text { Non-elderly, } \leq 64 \text { years } \\
(N=67)\end{array}$ & $\begin{array}{l}\text { Elderly, } \geq 65 \text { years } \\
(N=33)\end{array}$ & \\
\hline Age (years) & $52(19,64)$ & $69(65,84)$ & $<0.01$ \\
\hline Sex (male) & $46(68.7)$ & $22(66.7)$ & 0.84 \\
\hline \multicolumn{4}{|l|}{ Anthropometric measurements } \\
\hline $\operatorname{BMI}\left(\mathrm{kg} / \mathrm{m}^{2}\right)$ & $24.0(18.0,35.8)$ & $23.0(19.2,29.5)$ & 0.09 \\
\hline $\mathrm{BMI} \geq 25 \mathrm{~kg} / \mathrm{m}^{2}$ & $27(40.3)$ & $10(30.3)$ & 0.09 \\
\hline \multicolumn{4}{|l|}{ Waist circumference $(\mathrm{cm})$} \\
\hline Male & $86.0(63.9,105.5)$ & $89.9(74.5,99.0)$ & 0.53 \\
\hline Female & $82.0(66.7,114.0)$ & $73.0(70.0,94.5)$ & 0.12 \\
\hline \multicolumn{4}{|l|}{ Family history (present) } \\
\hline Diabetes mellitus & $41(61.2)$ & $17(51.5)$ & 0.36 \\
\hline Hypertension & $6(9.0)$ & $2(6.1)$ & 0.62 \\
\hline Cerebrovascular disease & $1(1.5)$ & $0(0.0)$ & 0.48 \\
\hline Heart disease & $1(1.5)$ & $0(0.0)$ & 0.48 \\
\hline \multicolumn{4}{|l|}{ Lifestyle characteristics } \\
\hline Alcohol consumption (everyday drinker) & $8(11.9)$ & $4(12.1)$ & 0.81 \\
\hline Smoking behavior (current smoker) & $20(29.9)$ & $6(18.2)$ & 0.24 \\
\hline \multicolumn{4}{|l|}{ Complications (present) } \\
\hline Diabetic neuropathy & $31(46.3)$ & $25(75.8)$ & $<0.01$ \\
\hline Diabetic retinopathy & $7(10.4)$ & $6(18.2)$ & 0.35 \\
\hline Diabetic nephropathy & $33(49.3)$ & $18(54.5)$ & 0.62 \\
\hline Cardiovascular complications & $37(55.2)$ & $15(45.5)$ & 0.36 \\
\hline Cerebrovascular complications & $42(62.7)$ & $16(48.5)$ & 0.18 \\
\hline \multicolumn{4}{|l|}{ Hypertension-related factors } \\
\hline Systolic blood pressure ( $\mathrm{mmHg}$ ) & $135(91,185)$ & $140(98,175)$ & 0.24 \\
\hline Diastolic blood pressure $(\mathrm{mmHg})$ & $85(47,107)$ & $72(47,95)$ & $<0.01$ \\
\hline Antihypertensive drug use (yes) & $18(26.9)$ & $19(57.6)$ & $<0.01$ \\
\hline \multicolumn{4}{|l|}{ Lipid-related parameters } \\
\hline $\begin{array}{l}\text { High-density lipoprotein cholesterol } \\
(\mathrm{mg} / \mathrm{dL})\end{array}$ & $53(28,116)$ & $56(29,114)$ & 0.87 \\
\hline $\begin{array}{l}\text { Low-density lipoprotein cholesterol } \\
(\mathrm{mg} / \mathrm{dL})\end{array}$ & $120.0(65.0,291.0)$ & $111.6(61.4,188.0)$ & 0.14 \\
\hline
\end{tabular}


Table 1 continued

\begin{tabular}{|c|c|c|c|}
\hline & \multicolumn{2}{|l|}{ Median (range) or $N(\%)$} & \multirow[t]{2}{*}{$P$ value } \\
\hline & $\begin{array}{l}\text { Non-elderly, } \leq 64 \text { years } \\
(N=67)\end{array}$ & $\begin{array}{l}\text { Elderly, } \geq 65 \text { years } \\
(N=33)\end{array}$ & \\
\hline Triglycerides (mg/dL) & $149(56,819)$ & $116(39,973)$ & 0.04 \\
\hline Antidyslipidemic drug use (yes) & $17(25.4)$ & $12(36.4)$ & 0.23 \\
\hline \multicolumn{4}{|l|}{ Diabetes-related items } \\
\hline Duration of diabetic treatment (years) & $7.5(0.5,24)$ & $12(0.5,35)$ & $<0.01$ \\
\hline Hemoglobin Alc (\%) at baseline & $10.8(8.0,17.1)$ & $9.6(8.1,13.9)$ & $<0.01$ \\
\hline \multicolumn{4}{|l|}{ Treatment of diabetes mellitus } \\
\hline Sulfonylurea & $28(41.8)$ & $22(66.7)$ & 0.02 \\
\hline Alpha glucosidase inhibitor & $10(14.9)$ & $6(18.2)$ & 0.68 \\
\hline Biguanides & $13(19.4)$ & $13(39.4)$ & 0.03 \\
\hline Thiazolidine derivatives & $17(25.4)$ & $11(33.3)$ & 0.40 \\
\hline DPP-4 inhibitor & $1(1.5)$ & $2(6.1)$ & 0.21 \\
\hline \multicolumn{4}{|l|}{ Number of oral glucose lowering drugs } \\
\hline 0 & $33(49.3)$ & $9(27.3)$ & 0.08 \\
\hline 1 & $11(16.4)$ & $4(12.1)$ & \\
\hline 2 & $11(16.4)$ & $11(33.3)$ & \\
\hline 3 & $12(17.9)$ & $8(24.2)$ & \\
\hline 4 & $0(0.0)$ & $1(3.0)$ & \\
\hline \multicolumn{4}{|l|}{ Type of insulin at initiation } \\
\hline Ultra-rapid insulin & $42(62.7)$ & $5(15.2)$ & $<0.01$ \\
\hline Rapid-acting insulin & $0(0.0)$ & $5(15.2)$ & $<0.01$ \\
\hline Mixed insulin & $16(23.9)$ & $4(12.1)$ & 0.17 \\
\hline Long-acting insulin & $14(20.9)$ & $20(60.6)$ & $<0.01$ \\
\hline Insulin dosage at initiation (U/day) & $18(8,34)$ & $10(6,24)$ & $<0.01$ \\
\hline
\end{tabular}

$P<0.05$ was considered statistically significant

The Mann-Whitney test for continuous variables and the Chi-square test for categorical variables were used to compare the two groups

$B M I$ body mass index

\section{Glycemic Control}

Changes from baseline in glycemic control, lipid profiles, body weight, and insulin dosage are shown in Tables 2 and 3. In the non-elderly patient group, HbA1c levels were significantly improved at $3(P<0.01), 6(P<0.01)$ and 12 months $(P<0.01)$ after initiation of insulin therapy, and reached $7.3 \%$ (range 6.0-12.0) at 12 months $\quad[-3.8 \% \quad(-11.4,0.9), \quad P<0.01]$. 


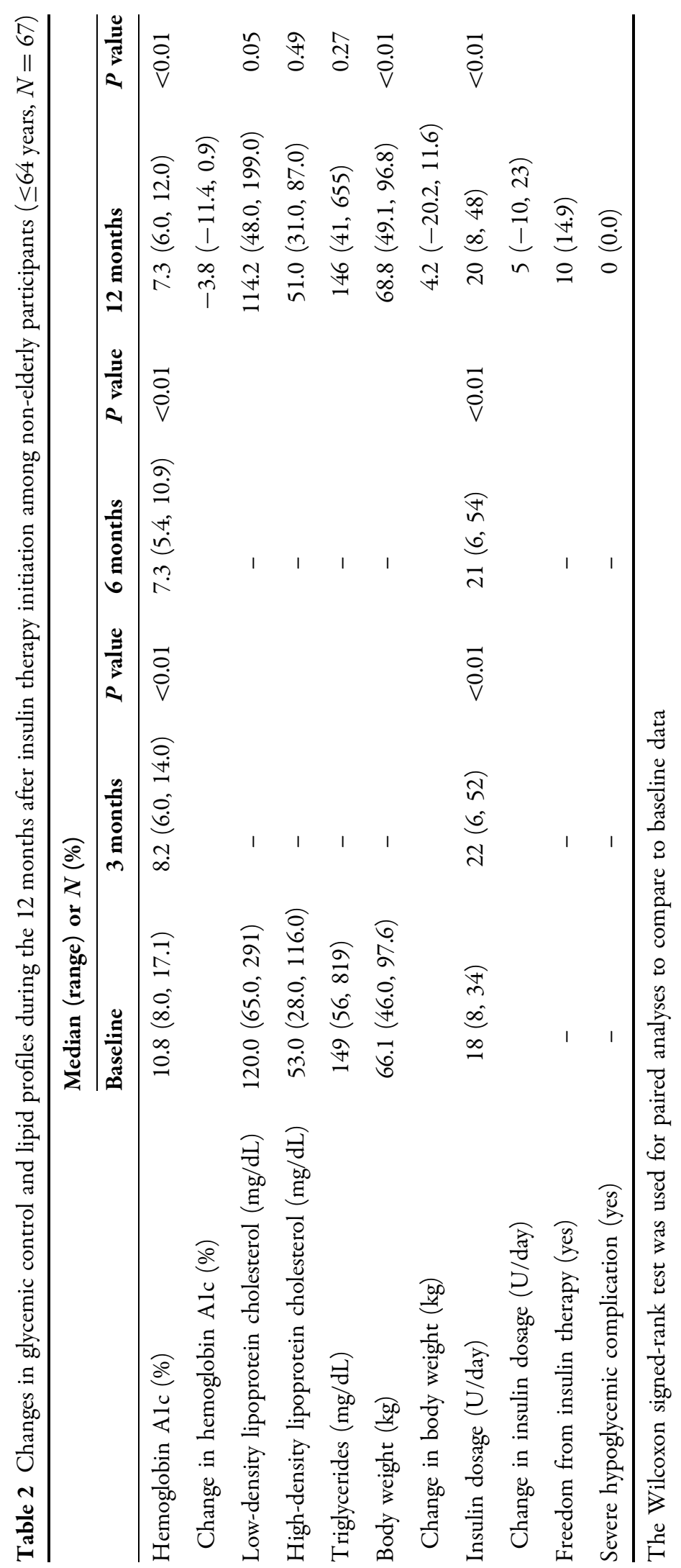




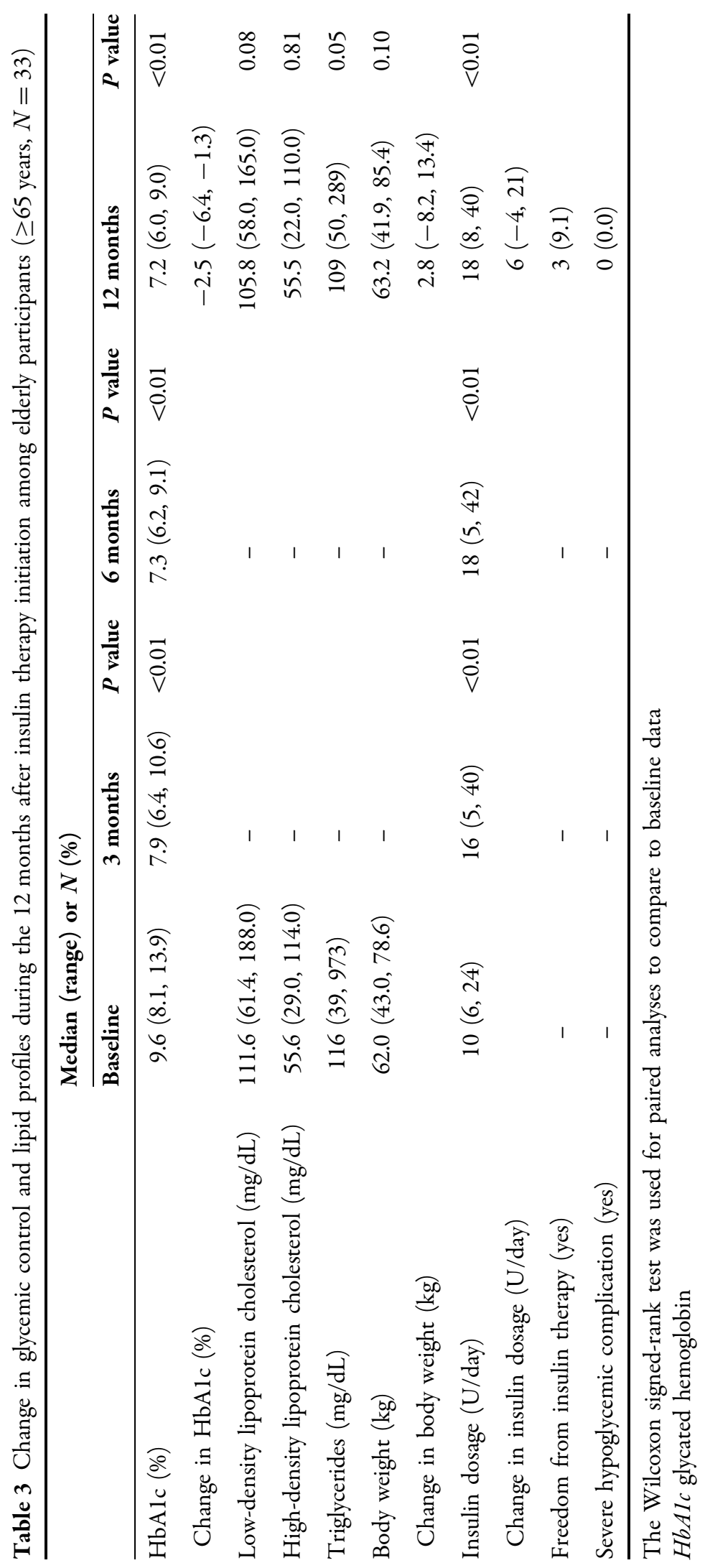


However, median insulin dosage [5 U/day $(-10$, 23)] and median body weight were significantly higher at 12 months $[4.2 \mathrm{~kg}(-20.2,11.6)$, $P<0.01$; Table 2]. For elderly patients, HbA1c levels were significantly improved at 3 $(P<0.01), \quad 6 \quad(P<0.01) \quad$ and 12 months $(P<0.01)$ after initiation of insulin therapy, and $\mathrm{HbA} 1 \mathrm{c}$ levels and reached [7.2\% $(6.0,9.9)$, $-2.5 \%(-6.4,-1.3), P<0.01]$ at 12 months. Insulin dosage increased [6 $(-4,21), P<0.01]$, but changes in body weight were not significant [2.8 (-8.2, 13.49), $P=0.10 ;$ Table 3]. Lipid profiles did not change significantly from baseline in either group at 12 months compared to baseline (Table 2).

Figure 1 shows HbA1c levels for elderly and non-elderly patients after a follow-up period of 1 year. After initiation of insulin therapy, diabetes remained uncontrolled (HbA1c $\geq 8 \%$ ) in $31.8 \%$ of non-elderly and $15.4 \%$ of elderly patients $(P=0.08)$. In the patient population overall, 26 patients $(26.0 \%)$ had uncontrolled diabetes and was more frequently in obese patients $\quad\left(\mathrm{BMI} \geq 25 \mathrm{~kg} / \mathrm{m}^{2}\right) \quad$ compared to patients with $\mathrm{HbA} 1 \mathrm{c}<8 \%$ after 1 year $(64.9 \%$ vs $76.9 \%, P=0.04$ ) (Table 4 ).

\section{DISCUSSION}

This retrospective cohort survey conducted at one medical clinic in Japan revealed the effectiveness of intensive insulin therapy for elderly Japanese patients ( $\geq 65$ years) with uncontrolled diabetes mellitus, demonstrating an improvement in glycemic control 1 year after initiation of insulin therapy that was comparable to that observed in non-elderly patients ( $<65$ years). In addition, severe hypoglycemic events were avoided in both age groups by comprehensive management using SMBG. This study suggests the potential for safely improving poor glycemic control among elderly patients as well as non-elderly patients by initiating insulin therapy. However, 37.8\% of non-elderly and $19.2 \%$ of elderly still experienced poor glycemic control

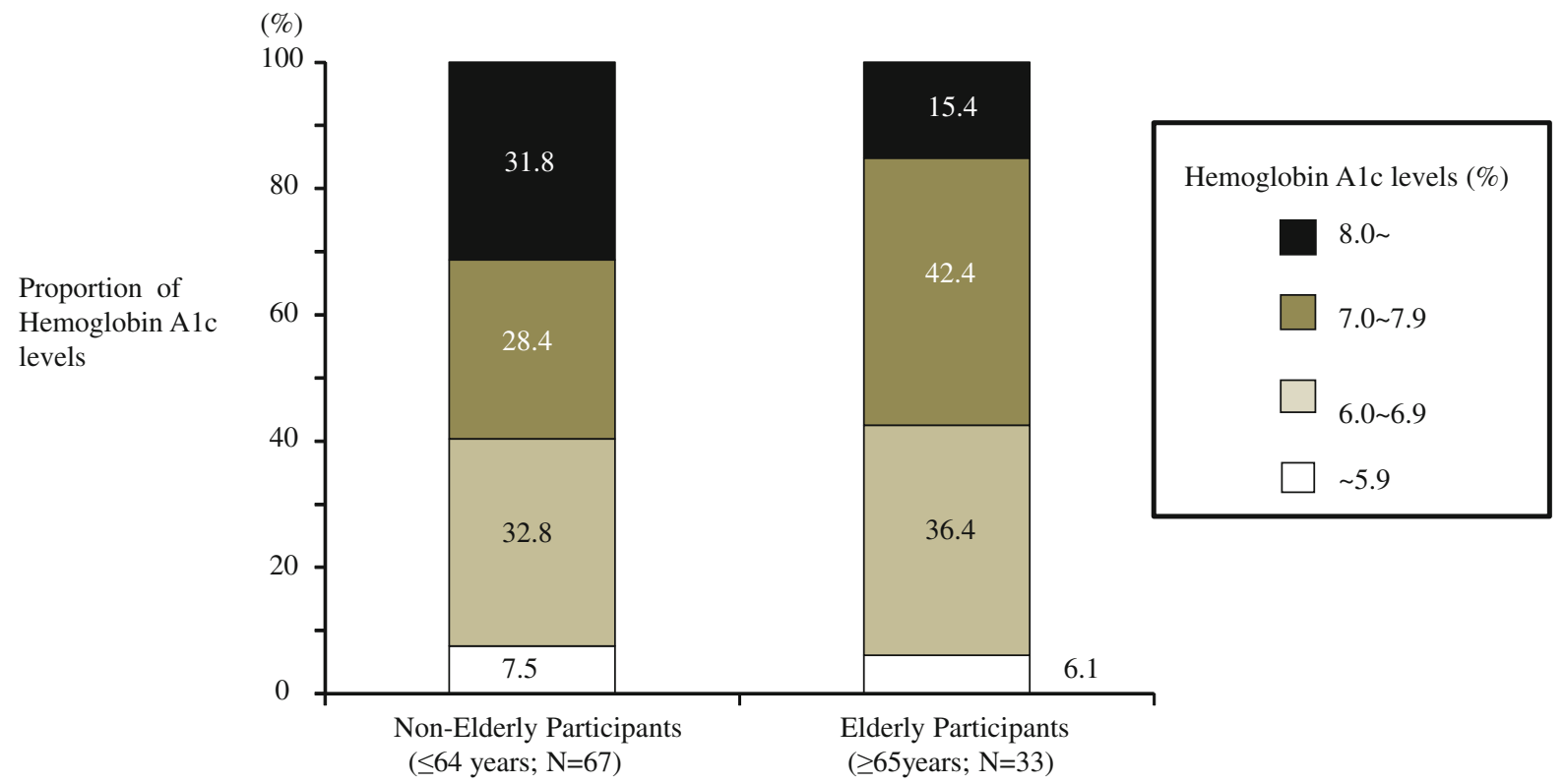

Fig. 1 Distribution of hemoglobin A1c (HbA1c) levels at 1-year follow-up 
(HbA1c $\geq 8.0 \%)$ after 1 year, and obesity was associated with poor glycemic control at 12 months in this study.

Although treatment strategies for elderly patients with diabetes mellitus are controversial, several intervention studies have reported the effectiveness of intensive treatment for elderly patients with diabetes $[14,15]$. A 6-year interventional observational study conducted in Japan reported an association between high HbA1c levels and the development of retinopathy, and that intensive control of diabetes mellitus led to favorable outcomes [14]. A study in Nagoya, Japan, found no increase in mortality among well-controlled elderly patients with diabetes compared to the general population after matching for age and sex [15]. Thus, better management of diabetes might prevent and/or delay diabetic complications or improve mortality, despite minimal accumulated evidence in the elderly.

In general practice, improving uncontrolled diabetes in elderly patients is sometimes more difficult than in non-elderly patients because of age-related deterioration in glucose tolerance and a reduction in endogenous insulin secretion, and therefore treatment is likely to require insulin initiation in elderly patients with uncontrolled glycemic control [16]. The Kumamoto study, a randomized controlled trial evaluating intervention with insulin therapy among 110 Japanese patients with uncontrolled type 2 diabetes, revealed the effectiveness of intensive treatment for primary and secondary prevention of diabetic complications, and showed that treatment with multiple insulin injections per day was more beneficial for total medical costs at 10 years of follow-up compared to a

Table 4 Baseline characteristics of participants with controlled and uncontrolled diabetes after 1-year follow-up

\begin{tabular}{|c|c|c|c|}
\hline & \multicolumn{2}{|l|}{ Median (range) or $N(\%)$} & \multirow[t]{2}{*}{$P$ value } \\
\hline & $\begin{array}{l}\text { Controlled group, } \\
\text { HbA1c }<8 \%(N=74)\end{array}$ & $\begin{array}{l}\text { Uncontrolled group, } \\
\text { HbA1c } \geq 8 \%(N=26)\end{array}$ & \\
\hline Proportion of elderly participants ( $\geq 65$ years) & $28(37.8)$ & $5(19.2)$ & 0.08 \\
\hline Sex (male) & $48(64.9)$ & $20(76.9)$ & 0.04 \\
\hline $\mathrm{BMI} \geq 25 \mathrm{~kg} / \mathrm{m}^{2}$ & $23(31.1)$ & $14(53.8)$ & 0.04 \\
\hline BMI $\left(\mathrm{kg} / \mathrm{m}^{2}\right)$ & $23.1(18.0,35.8)$ & $25.4(18.4,33.3)$ & 0.07 \\
\hline \multicolumn{4}{|l|}{ Lifestyle characteristics } \\
\hline Alcohol consumption (everyday drinker) & $11(30.6)$ & $1(7.7)$ & 0.10 \\
\hline Smoking behavior (current smoker) & $21(32.3)$ & $5(20.0)$ & 0.25 \\
\hline Antihypertensive drug use (yes) & $29(39.2)$ & $8(30.8)$ & 0.44 \\
\hline Antidyslipidemic drug use (yes) & $52(72.2)$ & $9(34.6)$ & 0.51 \\
\hline Diabetic complications (present) & $52(70.3)$ & $20(76.9)$ & 0.52 \\
\hline Macrovascular complications (present) & $56(74.0)$ & $17(65.4)$ & 0.31 \\
\hline
\end{tabular}

$P<0.05$ was considered statistically significant

The Mann-Whitney test for continuous variables and the Chi-square test for categorical variables were used to compare between controlled and uncontrolled group at 12 months

$B M I$ body mass index, HbAlc glycated hemoglobin 
conventional insulin injection therapy $[17$, 18]. Although these studies were conducted in middle-aged patients (mean age, 48.2 years in multiple injection therapy group), the results may be applicable to elderly patients. Thus, adequate insulin initiation and maintaining safe insulin therapy is necessary for improving uncontrolled diabetes, and have a beneficial effect on healthcare expenditure.

Several reports have brought attention to excessively strict glycemic control strategies. The Diabetes and Aging Study, a large-scale retrospective cohort study of 71,092 patients in the United States with diabetes mellitus, reported that mortality had a U-shaped relationship with $\mathrm{HbA1c}$, with an increased risk of all-cause mortality associated with HbA1c levels less than 6\% [19]. In the Health, Aging, and Body Composition Study, a prospective study conducted in the United States, insulin users with HbA1c levels $\leq 6.0 \%$ had a 4.36-fold higher risk of falls compared to those individuals with HbA1c levels $\geq 8.0 \%$ [8]. In addition to the above-mentioned influences on mortality and morbidity, hypoglycemic events can trigger a series of complications [8, 20]. In a large-scale cohort study in the United States, patients with diabetes (mean age, 64.0 years) who experienced hypoglycemic events were 1.79 times more likely to experience an acute cardiovascular events than those who did not experience hypoglycemic events; similar results were also reported for patients aged $\geq 65$ years compared to those for the entire population (odds ratio $=1.78$ ) [20]. In addition, the Action to Control Cardiovascular Risk in Diabetes (ACCORD) trial, which was a randomized trial among diabetic patients aged 40-79 years with cardiovascular histories or their risks, showed that the risk of severe hypoglycemia in an intensive glycemic control group (HbA1c goal $<6 \%$ ) was more than threefold higher than in a standard glycemic control group (HbA1c goal 7.0-7.9\%); furthermore, the mortality risk was 1.22 -fold higher in the intensive compared to the standard glycemic control group [7]. Therefore, avoidance of hypoglycemia is necessary for better glycemic control and to reduce mortality and morbidity. To avoid hypoglycemia in our study, we used SMBG, according to Japanese Diabetes Management Guidelines [21], which involved regularly checking patient $\mathrm{HbA1c}$ levels and SMBG records, and modifying their treatment accordingly. No severe hypoglycemic events were observed in either the elderly or non-elderly groups during the 1-year follow-up period. SMBG may allow patients to evaluate their glucose concentrations and assess their achievement status to target glycemic levels $[5,22]$. Therefore, comprehensive management that includes SMBG is a useful strategy for improving awareness and avoiding hypoglycemic events among insulin-treated elderly patients $[5,22]$. In addition, the use of long-acting insulin may also avoid the problem of hypoglycemia in elderly patients. The APOLLO study, comparing once-daily basal insulin glargine (long-acting insulin) versus thrice-daily prandial insulin lispro (shortacting insulin) in 418 patients with type 2 diabetes aged 18-75 years, showed that hypoglycemic events were lower in the glargine treatment group than in the insulin lispro group (4.27 vs 19.46 per person) [23]. On this basis it is therefore, important to consider the insulin type when initiating intensive insulin therapy in the elderly. To improve uncontrolled diabetes and prevent severe hypoglycemic events, treatment using insulin therapy can be acceptable as part of the comprehensive management for eligible patients who have the ability to use insulin therapy. 
Although glycemic control was safely improved after initiation of insulin therapy in this study, $31.8 \%$ of non-elderly and $15.4 \%$ of elderly participants still experienced uncontrolled diabetes with HbA1c levels of at least $8 \%$. Obese patients $(\mathrm{BMI} \geq 25)$ were significantly more likely to have uncontrolled diabetes. During the 1-year follow-up period, body weight was significantly higher versus baseline at 12 months among non-elderly participants (66.1 vs $68.8 \mathrm{~kg}, P<0.01$ ), but not among elderly participants $(62.0$ vs $63.2 \mathrm{~kg}$, $P=0.10)$. It is possible that elderly participants may demonstrate healthier lifestyle behaviors related to avoid weight gain after initiation of insulin therapy [3]. Obesity is a well-known risk factor for poor glycemic control, and the United Kingdom Prospective Diabetes Study (UKPDS $24)$, which was a randomized controlled trial among 4,075 patients aged 25-65 years with newly diagnosed type 2 diabetes including obese patients (48.2\%), showed that initiating insulin therapy induced more hypoglycemia and weight gain without necessarily providing better glycemic control [24]. Thus, both weight gain and hypoglycemia need to be addressed with adequate nutrition and exercise instruction.

There were several limitations to the present study. First, there may have been selection bias; the present study was conducted at one medical institution specializing in the treatment of diabetes. A large-scale multicenter study is needed to better compare our data to data from other medical settings. Second, this study did not include some important factors, in particular, social support, health behavior and nutrition status. Studies that obtain information regarding health behavior, nutrition status, and other related factors are needed. Third, our sample size was small. Similar to the first limitation, large-scale multicenter studies are required to address this issue. Fourth, differences existed in patient numbers, baseline HbA1c levels, type of insulin and dosage between the non-elderly and elderly patient groups. A large-scale study is required to adjust for these differences. Finally, 1 year is a relatively short study period. As a next step, cohort studies with long study periods (more than 5 years) are needed to assess long-term outcomes including glycemic control, onset of cardiovascular complications, mortality, and morbidity.

\section{CONCLUSION}

Our results indicate that newly initiated insulin therapy is as effective and safe for treating elderly Japanese patients with uncontrolled diabetes as for treating non-elderly patients. The study also clarified the utility of comprehensive management and education using SMBG to improve glycemic control and avoid hypoglycemia. The number of elderly patients with diabetes is expected to significantly increase not only in Japan, but worldwide too. Reducing morbidity and mortality requires improved glycemic control supported by adequate intensive management.

\section{ACKNOWLEDGMENTS}

We thank the staff of Taneda Medical Clinic for their excellent help in data collection. This study was funded by a 2012 Grant-in-Aid for Scientific Research (C) (No. 24590816).

Dr Hirohide Yokokawa is the guarantor for this article, and takes responsibility for the integrity of the work as a whole.

Conflict of interest. Takayoshi Shiga, Hirohide Yokokawa, Yoshinobu Taneda, 
Eiichiro Sugihara, Mayumi Meijyo, Kazunori Mitsuhashi, Teruhiko Hisaoka, and Hiroshi Isonuma declare no conflict of interest.

Compliance with ethics guidelines. This study was conducted according to the Ethical Guidelines for Epidemiological Studies established by the Japanese government, and with the Helsinki Declaration of 1975, as revised in 2000 and 2008. Informed consent was obtained from all patients for being in the study. The Ethics Committee of Juntendo University approved the research protocol (No. 832).

Open Access. This article is distributed under the terms of the Creative Commons Attribution Noncommercial License which permits any noncommercial use, distribution, and reproduction in any medium, provided the original author(s) and the source are credited.

\section{REFERENCES}

1. United Nations, Department of Economic and Social Affairs, Population Division. World population ageing: 1950-2050. Available from http://www.un.org/esa/population/publications/ worldageing19502050/. Accessed on 2 October, 2013.

2. Cabinet office, Government of Japan. Annual report on the aging society. 2013. Available from http:// www8.cao.go.jp/kourei/whitepaper/w-2013/zenbun/ 25pdf_index.html. Accessed on 1 September, 2013 (in Japanese).

3. Japanese Ministry of Health, Labour and Welfare. Health and Nutrition Survey 2011. Available from http://www.mhlw.go.jp/stf/houdou/2r9852000002 q1st-att/2r9852000002q1wo.pdf. Accessed on 2 October, 2013 (in Japanese).

4. Pogach LM, Brietzke SA, Cowan CL Jr, VA/DoD Diabetes Guideline Development Group, et al. Development of evidence-based clinical practice guidelines for diabetes: the Department of Veterans Affairs/Department of Defense guidelines initiative. Diabetes Care. 2004;27(Suppl 2):B82-9.
5. Inzucchi SE, Bergenstal RM, Buse JB, American Diabetes Association (ADA), European Association for the Study of Diabetes (EASD), et al. Management of hyperglycemia in type 2 diabetes: a patientcentered approach: position statement of the American Diabetes Association (ADA) and the European Association for the Study of Diabetes (EASD). Diabetes Care. 2012;35:1364-79.

6. Ali MK, Bullard KM, Saaddine JB, et al. Achievement of goals in US diabetes care, 1999-2010. N Engl J Med. 2013;368:1613-24.

7. Skyler JS, Bergenstal R, Bonow RO, et al. Intensive glycemic control and the prevention of cardiovascular events: implications of the ACCORD, ADVANCE, and VA diabetes trials: a position statement of the American Diabetes Association and a scientific statement of the American College of Cardiology Foundation and the American Heart Association. Diabetes Care. 2009;32:187-92.

8. Schwartz AV, Vittinghoff E, Sellmeyer DE, Health, Aging, and Body Composition Study, et al. Diabetes-related complications, glycemic control, and fall in older adults. Diabetes Care. 2008;31:391-6.

9. Whitmer RA, Karter AJ, Yaffe K, et al. Hypoglycemic episodes and risk of dementia in older patients with type 2 diabetes mellitus. JAMA. 2009;301:1565-72.

10. Martin S, Schneider B, Heinemann L, et al. Selfmonitoring of blood glucose in type 2 diabetes and long-term outcome: an epidemiological cohort study. Diabetologia. 2006;49:271-8.

11. Kashiwagi A, Kasuga M, Oka Y, The Committee on the Standardization of Diabetes Mellitus-Related Laboratory Testing, et al. International clinical harmonization of glycated hemoglobin in Japan: from Japan Diabetes Society to National Glycohemoglobin Standardization Program values. Diabetol Int. 2012;3:39-40.

12. Friedewald WT, Levy RI, Fredrickson DS, et al. Estimation of the concentration of low-density lipoprotein cholesterol in plasma, without use of the preparative ultracentrifuge. Clin Chem. 1972;18:499-502.

13. Ministry of Health, Labor and Welfare and Ministry of Education, Culture, Sports, Science and Technology. Ethical guideline for epidemiological studies. Available from http://www-bm.mhlw.go. jp/general/seido/kousei/i-kenkyu/ekigaku/0504sisin. html. 2008. Accessed on 2 October, 2013 (in Japanese).

14. Katakura M, Naka M, Kondo T, et al. Development, worsening, and improvement of diabetic 
microangiopathy in older people: six-year prospective study of patients under intensive diabetes control. J Am Geriatr Soc. 2007;55: 541-7.

15. Katakura M, Naka M, Kondo T, et al. Prospective analysis of mortality, morbidity, and risk factors in elderly diabetic subjects: Nagano study. Diabetes Care. 2003;26:638-44.

16. Basu R, Breda E, Oberg AL, et al. Mechanisms of the age-associated deterioration in glucose tolerance: contribution of alterations in insulin secretion, action, and clearance. Diabetes. 2003;52:1738-48.

17. Shichiri M, Kishikawa H, Ohkubo Y, et al. Longterm results of the Kumamoto Study on optimal diabetes control in type 2 diabetic patients. Diabetes Care. 2000;23(Suppl 2):B21-9.

18. Wake N, Hisashige A, Katayama $\mathrm{T}$, et al. Costeffectiveness of intensive insulin therapy for type 2 diabetes: a 10-year follow-up of the Kumamoto study. Diabetes Res Clin Pract. 2000;48:201-10.

19. Huang ES, Liu JY, Moffet $\mathrm{HH}$, et al. Glycemic control, complications, and death in older diabetic patients: the diabetes and aging study. Diabetes Care. 2011;34:1329-36.
20. Johnston SS, Conner C, Aagren M, et al. Evidence linking hypoglycemic events to an increased risk of acute cardiovascular events in patients with type 2 diabetes. Diabetes Care. 2011;34:1164-70.

21. Japan Diabetes Society. Treatment guideline for diabetes 2012-2013. Tokyo: Bunkodo Co, Ltd. 2012 (in Japanese).

22. Boutati EI, Raptis SA. Self-monitoring of blood glucose as part of the integral care of type 2 diabetes. Diabetes Care. 2009;32(Suppl 2):S205-10.

23. Bretzel RG, Nuber U, Landgraf W, et al. Once-daily basal insulin glargine versus thrice-daily prandial insulin lispro in people with type 2 diabetes on oral hypoglycaemic agents (APOLLO): an open randomised controlled trial. Lancet. 2008;29(371): 1073-84.

24. United Kingdom Prospective Diabetes Study 24. A 6-year, randomized, controlled trial comparing sulfonylurea, insulin, and metformin therapy in patients with newly diagnosed type 2 diabetes that could not be controlled with diet therapy. United Kingdom Prospective Diabetes Study Group. Ann Intern Med. 1998;128:165-75. 